\title{
Genome-wide analysis reveals four key transcription factors associated with cadmium stress in creeping bentgrass (Agrostis stolonifera L.)
}

Jianbo Yuan ${ }^{1,2}$, Yuqing Bai ${ }^{3}$, Yuehui Chao ${ }^{2}$, Xinbo Sun ${ }^{4}$, Chunyan He ${ }^{2}$, Xiaohong Liang ${ }^{2}$, Lijuan Xie ${ }^{\text {Corresp. }}$ 1, Liebao Han Corresp. 2

${ }^{1}$ School of Applied Chemistry and Biotechnology, Shenzhen Polytechnic, Shenzhen, China

2 Turfgrass Research Institute, College of Forestry, Beijing Forestry University, Beijing, China

3 Administrative Office, Wutong Mountain National Park, Shenzhen, China

4 Key laboratory of crop growth regulation of Hebei Province, Hebei Agricultrual University, China

Corresponding Authors: Lijuan Xie, Liebao Han

Email address: xlj@szpt.edu.cn, hanliebao@163.com

Cadmium (Cd) toxicity seriously affects the growth and development of plants, so studies on uptake, translocation, and accumulation of $\mathrm{Cd}$ in plants are crucial for phytoremediation. However, the molecular mechanism of the plant response to $\mathrm{Cd}$ stress remains poorly understood. The main objective of this study was to reveal differentially expressed genes (DEGs) under lower (BT2_5) and higher (BT43) Cd concentration treatments in creeping bentgrass. A total of 463,184 unigenes were obtained from creeping bentgrass leaves using RNA sequencing technology. Observation of leaf tissue morphology showed that the higher $\mathrm{Cd}$ concentration damages leaf tissues. Four key transcription factor (TF) families, WRKY, bZIP, ERF, and MYB, are associated with Cd stress in creeping bentgrass. Our findings revealed that these four TFs play crucial roles during the creeping bentgrass response to $\mathrm{Cd}$ stress. This study is mainly focused on the molecular characteristics of DEGs under Cd stress using transcriptomic analysis in creeping bentgrass. These results provide novel insight into the regulatory mechanisms of respond to $\mathrm{Cd}$ stress and enrich information for phytoremediation. 
4 Jianbo Yuan $1,2,{ }^{*}$, Yuqing Bai ${ }^{*}$, Yuehui Chao ${ }^{2}$,Xinbo Sun ${ }^{4}$,Chunyan $\mathrm{He}^{2}$, Xiaohong Liang 2 , Lijuan

$5 \quad \mathrm{Xie}^{1}$ and Liebao $\operatorname{Han}^{2}$

6 1School of Applied Chemistry and Biotechnology, Shenzhen Polytechnic, 518055, Shenzhen, China

$7 \quad$ 2Turfgrass Research Institute, College of Forestry, Beijing Forestry University, Beijing, China

83 Administrative Office, Wutong Mountain National Park, 518004, Shenzhen, China

9 4Key labroratory of crop growth regulation of Hebei Province, Hebei Agricultural University, Baoding,

10 071001, Baoding, China

$11 *$ These authors contributed equally to this work. Corresponding

12 Authors: Lijuan Xie, Liebao Han

13 Email address: xlj@szpt.edu.cn, hanliebao@163.com

\section{ABSTRACT}

15 Cadmium $(\mathrm{Cd})$ toxicity seriously affects the growth and development of plants, so studies on uptake,

16 translocation, and accumulation of $\mathrm{Cd}$ in plants are crucial for phytoremediation. However, the molecular

17 mechanism of the plant response to Cd stress remains poorly understood. The main objective of this study was

18 to reveal differentially expressed genes (DEGs) under lower (BT2_5) and higher (BT43) Cd concentration

19 treatments in creeping bentgrass. A total of 463,184 unigenes were obtained from creeping bentgrass leaves

20 using RNA sequencing technology. Observation of leaf tissue morphology showed that the higher $\mathrm{Cd}$

21 concentration damages leaf tissues. Four key transcription factor (TF) families, WRKY, bZIP, ERF, and MYB,

22 are associated with Cd stress in creeping bentgrass. Our findings revealed that these four TFs play crucial roles

23 during the creeping bentgrass response to $\mathrm{Cd}$ stress. This study is mainly focused on the molecular

24 characteristics of DEGs under Cd stress using transcriptomic analysis in creeping bentgrass. These results

25 provide novel insight into the regulatory mechanisms of respond to $\mathrm{Cd}$ stress and enrich information for 26 phytoremediation.

\section{INTRODUCTION}


28 Cadmium pollution is an environmental issue, which is getting more and more attention. As a non-essential,

29 highly toxic and easily accumulated element in plants, Cd accumulation directly inhibit plant development by

30 negative effects on physiological and metabolic processes (Nawrot et al., 2006; Hasan et al., 2009; DalCorso et

31 al., 2010). Soil contamination with $\mathrm{Cd}$ has long been a major ecological concern worldwide in areas experiencing industrialization and urbanization (Chen al., 2011; Yu et al., 2017).

There are two main strategies for fighting Cd pollution, phytoextraction and phytostabilization (Salt et al., 1998). Phytoextraction involves applying a cover of vegetation at the contaminated site, thereby preventing wind and water erosion while also developing an extensive root system that will fix soil. This method requires plants that possess tolerance to the contaminant metals and strong soil-fixing abilities. Phytostabilization employs species from the plant communities found on local contaminated sites (Krämer et al., 2005). In addition, breeding of cultivars that rapidly accumulate $\mathrm{Cd}$ is useful for reducing $\mathrm{Cd}$ pollution. For these purposes, understanding the molecular mechanisms of plant responses to $\mathrm{Cd}$ stress is critically important. characteristic provides a powerful benefit for phytoremediation. Several previous studies have suggested that certain fast-growing, high-biomass, and metal-tolerant plant species may be effective for phytoremediation (Juwarkar et al., 2008). Among the molecular mechanisms of plants related to Cd stress, Satoh-Nagasawa et al. (2011) found that $O S H M A 2$ is the major transporter of $\mathrm{Cd}$ from roots to shoots in rice. OsNRAMP1 participates in Cd uptake and transport at the cellular level in rice, and OsNRAMPl overexpression in roots may promote Cd accumulation in the shoots (Takahashi et al., 2011). OsHMA3 is critical for $\mathrm{Cd}$ stress in rice, and mutation of this protein results in loss of $\mathrm{Cd}$ function into vacuoles in root cells, leading to high translocation of $\mathrm{Cd}$ from roots to shoots (Miyadate et al., 2011; Ueno et al., 2010; Takahashi et al., 2011). OsNRAMP5 encodes a natural resistance-associated macrophage protein in rice, and functional analysis showed that a defect in this protein decreases Cd uptake by roots through the use of a mutant method in Arabidopsis thaliana (Ishikawa et al., 2012). Moreover, several transcription factors (TFs) in several families have been identified in the Cd stress response, including WRKY, ERF, MYB, and bZIP (Wei et al., 2008; Tang et al., 2005; van de Mortel et al., translocation, and accumulation of $\mathrm{Cd}$ in plants. 
57 has provided a powerful approach for transcriptomic and non-coding RNA research (Ekblom et al., 2011). 58 Zhang et al. (2009) have suggested that RNA-Seq can be applied to multiple total or fractionated RNA 59 molecules, which are converted to a library of complementary DNA (cDNA) fragments and all molecules are 60 sequenced with a high-throughput method to obtain short sequence reads. Recently, RNA-Seq has been widely 61 applied to analysis of differential gene expression in plant stress responses, for example, Cd stress in radish (Xu 62 et al., 2015). The purpose of this study was to identify differentially expressed genes (DEGs) in creeping 63 bentgrass under $\mathrm{Cd}$ stress conditions for further analysis.

64 Creeping bentgrass is widely used on golf courses and for urban landscaping. Previous research on this plant has mainly focused on the physiological level, such as the effects of chelated iron, biostimulants, and nitrogen on the growth of creeping bentgrass (Ervin et al., 2004; Snyder, 1972). However, the molecular mechanism of the bentgrass response to $\mathrm{Cd}$ stress remains unclear. In this study, we isolated a total of 49.6, 52.2, and 50.9 million clean reads from CK, BT2_5, and BT43 creeping bentgrass leaf cDNA libraries, respectively, using RNA-Seq methods. Our study investigated the molecular characteristics of $\mathrm{Cd}$ stress response in creeping bentgrass.

\section{MATERIALS AND METHODS}

\section{Plant material preparation}

73 The cultivar of creeping bentgrass used in this study was A4, obtained from the forestry college of Beijing

74 Forestry University. The seeds were soaked in water for 3 days. Then, seeds were sown into soil consisting of 75 peat, vermiculite, and perlite at a ratio of $1: 1: 1$, and containing no foreign $\mathrm{Cd}$. The seedlings were grown in a 76 greenhouse at $26^{\circ} \mathrm{C}$ and plant materials used in this study were mature plants of 3-month old irrigated with 77 Hoagland nutrient solution (Hoagland and Arnon, 1950). For Cd stress treatments, plant materials were 78 irrigated with nutrient solution every 2 days in which $2.5 \mathrm{mM}$ (BT2_5) or $43 \mathrm{mM}$ (BT43) $\mathrm{CdCl}_{2}$ was added. 79 Plant samples (aboveground) were harvested under treatment for 7 days. Samples were frozen in liquid 80 nitrogen and stored at $-80^{\circ} \mathrm{C}$ for further experiments. All samples were taken as three biological replicates.

\section{Leaf tissue morphology observation}

82 We selected CK, BT2_5, and BT43 leaves for cutting. Fresh plant tissue was placed in 4\% paraformaldehyde 83 solution for $36 \mathrm{~h}$, followed by $4 \mathrm{~h}$ of dehydration using $75 \%$ ethanol, and $4 \mathrm{~h}$ of dehydration using $90 \%$ and $8495 \%$ ethanol. Then the plant material was converted into a paraffin section using a microtome. The thickness of 
85 the slice was $4 \mu \mathrm{m}$, and it was stained with saffron and fast green FCF. Finally, the slice was used for light

86 microscope observation (Nikon ECLIPSE-ci).

87 Plant RNA isolation and cDNA preparation

88 Three cDNA libraries were constructed for RNA sequencing. Total RNA of CK, BT2_5, and BT43 were

89 extracted using extraction kits (TIANGEN, Beijing, China). The First Strand cDNA Synthesis Kit as well as the

90 PrimeScript $^{\mathrm{TM}}$ RT Reagent Kit with gDNA Eraser (Takara, Beijing, China) were used. The reverse

91 transcriptional reaction used $1 \mu$ of total RNA.

92 Sequencing, data filtering, and transcript assembly

93 The CK, BT2_5, and BT43 cDNA libraries were used for high-throughput sequencing (Illumina HiSeq ${ }^{\mathrm{TM}}$ ), and

94 raw reads were obtained. After verifying the quality of all raw reads, the $\mathrm{Q}_{\text {phred }}$ value was determined,

95 representing base quality in four grades at 90\% (Q10), 99\% (Q20), 99.9\% (Q30), and 99.99\% (Q40). Raw

96 reads were filtered to remove adapters, uncertain base identifications, and $\mathrm{Q}_{\text {phred }}$; no more than 20 reads were

97 removed. We obtained clean reads by filtering, which were then used for assembly using Trinity (Grabherr et

98 al., 2011). After the transcript sequence was obtained, transcript sequences were arranged into clusters by

99 comparison using Corset (https://code.google.com/p/corsetproject/). Finally, transcript and cluster sequence

100 lengths were used for analysis.

\section{Annotation of DEGs}

102 The clean reads were mapped in RSEM software (Li et al., 2011) to obtain read counts. From the read counts,

103 the density distribution was determined using fragments per kilobase of transcript per million mapped reads

104 (Trapnell et al., 2010), with an E-value threshold of greater than 0.3. DEGs were identified using DESeq

105 analysis (Anders and Huber, 2010). The padj threshold was no more than 0.05. Then, volcano plots and Venn

106 diagrams were used for DEG analysis, and the K-means and SOM methods were used for clustering DEGs.

\section{Gene functional analysis and classification}

108 To fully understand the functions of unigenes, a bioinformatics approach was used to annotate the unigenes.

109 First, sequences were aligned based on four data libraries, including the NCBI non-redundant protein sequences

110 (Nr), NCBI nucleotide sequences (Nt), Protein family (Pfam), and Swiss-Prot databases. The E-value 111 thresholds of $\mathrm{Nr}$ (diamond v0.8.22), Nt (NCBI blast 2.2.28+), and Swiss-Prot (diamond v0.8.22) were 1e-5, and

112 the E-value threshold of Pfam (HMMER 3.0) was 0.01. Second, based on the results of the Nr and Pfam

113 annotation, Gene Ontology (GO) was used to analyze unigene functions (Götz, et al., 2008), and the E-value 
114 threshold for GO (Blast2GO v2.5) was 1e-6. Finally, the Kyoto Encyclopedia of Genes and Genomes (KEGG)

115 and eukaryotic ortholog groups (KOG) were used to further annotate unigene functions. The E-value threshold

116 used for KEGG (KEGG Automatic Annotation Server) was 1e-10 and KOG (diamond v0.8.22) was 1e-3.

117 Real-time quantitative polymerase chain reaction (qRT-PCR) validation

118 Total RNA from CK, BT2_5, and BT43 were extracted using the method described above following qRT-PCR

119 analysis. The method used for first strand cDNA synthesis was the same as described above, with primers 120 designed using Primer Premier 5.0 (Premier Biosoft International, Palo Alto, CA, USA), shown in Table S13.

121 18S RNA was used as an internal control. The BioRadC1000Server system was used to perform qRT-PCR. The 122 reaction procedure was: 40 cycles of $95^{\circ} \mathrm{C}$ for $10 \mathrm{~min}, 95^{\circ} \mathrm{C}$ for $15 \mathrm{~s}$, and $60^{\circ} \mathrm{C}$ for $1 \mathrm{~min}$, and then melting 123 curve data was obtained. All reactions were run with technical duplicates. The $2^{-\Delta \Delta C t}$ method was used to 124 calculate relative expression levels (Livak et al., 2001).

\section{RESULTS}

\section{Leaf tissue morphological analysis under Cd stress}

127 To elucidate the effect of $\mathrm{Cd}$ treatment at various concentrations on leaf cellular, histological sections were 128 observed using a light microscope, as shown in Figure 1. The histological results revealed that the 129 morphological characteristics of the leaves changed markedly under the CK, BT2_5, and BT43 conditions. CK 130 cells were morphologically normal, complete, and healthy, whereas the morphology of cells treated with BT2_5 131 and BT43 was anomalous. Both BT2 5 and BT43 cells were damaged, and BT43 exhibited a greater degree of 132 damage than BT2_5. These histological observations suggested that Cd toxicity affected the morphological of 133 plant tissues, and that higher concentrations of Cd caused more serious effects.

\section{RNA sequencing and transcriptome assembly}

135 CK, BT2_5, and BT43 leaves were selected for RNA sequencing. All data were generated using three 136 biological replicates. RNA sequencing data quality is shown in Table 1. More than 50 million raw reads were 137 generated for each sample, and after filtering more than 49 million clean reads remained. The false discovery 138 rate of all data is less than or equal to 0.02 . All clean raw data were assembled. The transcript and unigene

139 length distribution is shown in Figure 2. The mean transcript length was 473 bases, and the mean unigene 140 length was 466 bases. Transcript N50 was 533 bases and unigene N50 was 466 bases (Figure 2a, b).

\section{Gene functional annotation and classification}

142 All-unigenes were searched for annotations against various databases. From a total of 463,184 sequences 
143 retrieved from different databases listed in Table 2, 203,561 were annotated in NR (43.94\%). The mapping rate

144 of NCBI nucleotide sequences (NT) was 167,084 (36.7\%). A total of 111,508 sequences were annotated in

145 Swiss-Prot (24.07\%) and 27,394 transcripts were mapped in the KOG databases into 26 categories, shown in

146 table S1. Most sequences clustered into 'Posttranslational modification, protein turnover, chaperones', which

147 accounted for $14.41 \%(4,431)$, followed by 'General function prediction only' $(3,402,11.06 \%)$, 'Translation,

148 ribosomal structure and biogenesis' (3037, 9.87\%), and 'Intracellular trafficking, secretion, and vesicular

149 transport' $(2,166,7.04 \%)$. The 'Unnamed protein' cluster was the least represented group, with only one 150 gene included.

151 GO assignments were used to classify the gene functions of assembled transcripts from creeping

152 bentgrass using Blast2GO. In all, 556,359 transcripts were mapped, and the GO databases provided 56

153 terms at the second level (Figure S1). The 'cellular process' term represented the largest cluster in the

154 biological process category, accounting for $11.09 \%(61,687)$, followed by 'metabolic process' $(60,277$,

$15510.83 \%)$ and 'single-organism process' (43,345, 7.79\%). In the cellular component, 'cell' (31,410, 5.65\%),

156 'cell part' $(31,387,5.64 \%)$, and 'macromolecular complex' $(18,918,3.4 \%)$ were the most abundant

157 categories. The 'binding' term was most represented within the molecular function category, accounting

158 for $12.57 \%(69,935)$, followed by 'catalytic activity' (51940, 9.34\%) and 'transporter activity' (5904,

159 1.06\%). Moreover, 'transcription factor activity, protein binding', 'transporter activity', 'response to

160 stimulus', and 'negative regulation of biological process' were also identified in creeping bentgrass under

161 Cd stress. Detailed GO data are shown in Tables S2 and S3.

162 KEGG was also used to identify the gene biological pathways. The assembled transcripts were

163 searched against the KEGG database (http://www.genome.jp/kegg/) with an E-value cutoff of 1e-10.

164 Overall, 48,400 transcripts were involved in 130 KEGG pathways (Table S3). All KEGG terms at the 165 second level are shown in Figure 3. 'Carbohydrate metabolism' was the highest category, accounting for

$16612.18 \%$ (5895). In addition, several categories exhibited a high ratio, such as 'Environmental adaptation'

167 (3430, 7.09\%), 'Folding, sorting and degradation' (4087, 8.44\%), and 'Transport and catabolism' (2633,

$1685.44 \%$ ). These categories may play important roles in response to Cd stress.

\section{Functional annotation of DEGs involved in Cd stress}

170 In the present study, clean reads from the CK, BT2_5, and BT43 libraries were mapped to transcriptome 171 reference sequences using DESeq (Anders and Huber, 2010). Unigenes that met the screening thresholds 
172 of $\left|\log _{2} \mathrm{FC}\right|>1$ and $\mathrm{p}<0.005$ were confirmed as DEGs. Comparison of the CK and BT2_5 libraries

173 indicated that 90 DEGs were upregulated and 189 DEGs were downregulated (Figure 4a), while 1175

174 DEGs were upregulated and 1334 DEGs were downregulated between the BT2_5 and BT43 libraries

175 (Figure 4b). Moreover, comparing CK with BT43 showed that 1730 DEGs were upregulated and 1761

176 DEGs were downregulated (Figure 4c).

177 GO enrichment analysis was performed to obtain functional annotations of the DEGs. Comparison 178 of CK with the BT2_5 library exhibited that 'catalytic activity (GO: 0003824)' was the predominant GO 179 term in the molecular function category, including 10 upregulated genes. The dominant GO term in the 180 biological process group was 'metabolic process (GO: 0008152)', which included 14 upregulated genes.

181 The 'membrane (GO: 0016020)' GO term was predominant among cellular components, including seven 182 upregulated genes (Table S3). In the BT2_5 and BT43 library comparison, the predominant GO terms 183 included 'metabolic process (GO: 0008152, 414)' in biological processes, 'catalytic activity (GO: $1840003824,385)$ ' in molecular functions, and 'membrane (GO: 0016020, 135)' in cellular components

185 (Table S3). Comparing BT43 with CK showed that the predominant GO terms were 'metabolic process 186 (GO: 0008152, 592)' in biological processes, 'catalytic activity (GO: 0003824, 556)' in molecular 187 functions, and 'cell (GO: 0005623, 186)' in cellular components (Table S3). Among the upregulated GO 188 terms, the predominant GO terms between CK and BT2_5 were 'metabolic process (GO: 0008152, 59)' in 189 the biological process group, 'binding (GO: 0005488, 55)' in the molecular function group, and 'cell (GO: 190 0005623, 30)' in the cellular component category (Table S3). Comparing the BT43 and BT2_5 libraries 191 indicated that the GO terms 'metabolic process (GO: 008152, 419)' in biological processes, 'catalytic 192 activity (GO: 0003824, 389)' in molecular functions, and 'membrane (GO: 0016020, 113)' in cellular 193 components were predominant (Table S3). The 'metabolic process (GO: 0008152, 634)' term in biological 194 processes, 'catalytic activity (GO: 0003824, 575)' in molecular functions, and 'cell (GO: 0005623, 278)' 195 in cellular components dominated the comparison between CK and BT43 (Table S3). In addition, the

196 'metal ion binding', 'transport', and 'UDP-glycosyltransferase activity' terms were also active during Cd 197 stress in creeping bentgrass.

198 To further determine how these DEGs are involved in biological metabolic pathways, KEGG 199 analysis was implemented to identify pathways associated with Cd stress using the hypergeometric test 200 method. In all, 152 pathways were upregulated and 182 pathways were downregulated among various 
201 libraries. Comparing CK and BT2_5 showed that the 'Galactose metabolism (ko00052)' and 'Starch and 202 sucrose metabolism (ko00500)' terms were predominant among upregulated pathways (Table S4), while 203 the 'Plant-pathogen interaction (ko04626)', 'Cutin, suberin and wax biosynthesis (ko00073)', and 204 'Peroxisome (ko04146)' terms accounted for most genes in downregulated pathways (Table S5). In the 205 BT2_5 and BT43 libraries (Table S4, Table S5), the predominant upregulated pathways were 'Glutathione 206 metabolism (ko00480)', 'Plant hormone signal transduction (ko04075)', and 'Phenylpropanoid

207 biosynthesis (ko00940)', and the predominant downregulated pathways were 'DNA replication 208 (ko03030)', 'Phenylpropanoid biosynthesis (ko00940)', and 'Isoquinoline alkaloid biosynthesis 209 (ko00950). Comparison of BT43 with CK showed that 'Starch and sucrose metabolism (ko00500)',

210 'Galactose metabolism (ko00052)', and 'Plant hormone signal transduction (ko04075)' hold predominant 211 positions in upregulated pathways (Tables S4 and S5), whereas the predominant positions in 212 downregulated pathways were 'Photosynthesis - antenna proteins (ko00196)', 'Starch and sucrose 213 metabolism (ko00500)', and 'Galactose metabolism (ko00052)'. In addition, several pathways were also 214 activated in creeping bentgrass under Cd stress, such as 'Glutathione metabolism (ko00480)', 'RNA 215 degradation (ko03018)', and 'ABC transporters (ko02010)'. These pathways were important for creeping 216 bentgrass resistance to Cd stress.

217 Several functional genes are involved in signal transduction pathways

218 Mitogen-activated protein kinase (MAPK) pathways represent a signaling mechanism in the plant 219 response to $\mathrm{Cd}$ stress that includes MAPK, MAPK kinase (MAPKK), and MAPK kinase kinase 220 (MAPKKK). The MAPK cascade plays critical roles in the plant response to Cd stress. Under Cd stress, 221 many kinases must be activated, and these kinase enzymes belong to the MAPK family. In the present 222 study, MAPKKK3 and MAPKKK12 were involved in the bentgrass response to Cd stress. In the CK to 223 BT43 comparison, MAPKKK3 was downregulated (Table S6). On the other hand, MAPKKK12 was 224 upregulated from CK to BT43.

225 Glutathione S-transferase (GST) is important for plant adaptation to a variety of stressful 226 conditions, because GST promotes the scavenging of reactive oxygen species (ROS). In our study, several 227 GSTs were obtained, including GSTU17, GSTU6, GSTU22, GST3, GST4, and GST23. GSTU17 was 228 downregulated from CK to BT2_5, whereas it was upregulated from BT2_5 to BT43 (Table S7). The other 229 'GSTU-' enzymes were consistent with GSTU17. In addition, GST4 and GST23 were upregulated in all 
230 RNA-Seq libraries. Salicylic acid (SA) is important in a well-known hormonal signal transduction

231 pathway related to metal stress. SA can provide plants with the protective ability to resist unfavorable

232 conditions. Our results indicated that salicylic acid-binding protein (SABP)-2 was active, as SABP2 was

233 upregulated in all libraries (Table S8). In summary, some signal transduction pathways play critical roles

234 in the creeping bentgrass response to Cd stress.

235 WRKY TFs involved in Cd stress

236 The WRKY gene family in plants contains specific transcriptional regulators, which play important roles

237 in plant responses to stress. WRKY proteins bind W-boxes to regulate many stress-related genes (Eulgem

238 et al., 1999). In our study, several WRKY TFs exhibited significant differential expression under the

239 different $\mathrm{Cd}$ treatment conditions, including WRKY33, WRKY12, WRKY23, WRKY75, WRKY2, and

240 WRKY53 (Table S9). In the BT2_5 treatment, WRKY33 was downregulated, but was upregulated

241 following BT43 treatment. WRKY12 and WRKY2 were also upregulated following BT2_5 treatment, but

242 these TFs were downregulated in the BT43 treatment concentration. WRKY23 and WRKY75 were

243 upregulated under all treatment conditions. On the other hand, WRKY53 was downregulated in all

244 treatments. KEGG pathway enrichment exhibited that WRKY33 and WRKY2 were involved in 'Plant-

245 pathogen interaction' pathway (ko04626). The role of WRKY33 is to suppress the induction of defense

246 genes. The role of $W R K Y 2$ is to promote defense related-gene and accelerate programmed cell death.

247 Response of basic leucine zipper motif (bZIP) TFs to Cd stress in bentgrass

248 The leucine zipper dimerization motif (Box 1) is a basic region found in the bZIP family. The bZIP TFs

249 play important roles in stress signal transduction. RNA-Seq results indicated that three DEGs belonging to

250 the bZIP family are involved in Cd stress in bentgrass, including basic leucine zipper 6 (BZIP06), BZIP43,

251 and BZIP19. All bZIP TFs were upregulated in the BT2_5 treatment, but BZIP06 and BZIP19 were

252 downregulated in the BT43 treatment (Table S10). Only BZIP43 maintained upregulation in BT43.

253 Ethylene-responsive factor (ERF) TFs related to Cd stress

254 The ethylene-responsive factor (ERF) subfamily belongs to the APETALA 2 (AP2) family, which

255 responds to abiotic stresses in plants, including ethylene, drought, and high salinity. In our study, several

256 ERF genes were observed under Cd stress in bentgrass, including ERF1B, ERF115, ERF110, ERF7,

$257 E R F 113, E R F 4$, and ERF15. In the BT2_5 Cd treatment, most genes were upregulated, while ERF115 and

258 ERF4 were slightly downregulated. In the BT43 treatment, most genes were upregulated, including 
$259 E R F 1 B, E R F 115$, and ERF15. The $\log _{2}$ ratios of these genes exhibited more than 5-fold changes. Only

260 ERF4 was slightly downregulated in BT43 (Table S11). KEGG pathway enrichment showed that ERF1B

261 was involved in 'Ethylene' pathway (ko04075) and ERF1B plays an important regulatory role in

262 promoting senescence.

263 MYB TF responses to Cd stress

264 MYB TFs are key factors in the regulation of development, metabolism, and responses to abiotic stresses

265 in plants. The RNA-Seq results indicated that four DEGs in the MYB family were involved in the Cd

266 stress response in bentgrass, including MYB4, MYB39, MYB108, and MYB305. Most of these genes were

267 upregulated in the BT2_5 and BT43 libraries (Table S12). Only MYB4 was downregulated in BT_25, but

268 this gene was upregulated in BT43.

269 Validation of DEGs via qRT-PCR

270 To confirm the differential expression patterns of genes identified in the RNA-Seq data, nine candidate

271 genes were randomly selected for qRT-PCR. The expression level of each gene from CK, BT2_5, and

272 BT23 was compared with its abundance based on the RNA-Seq data. The results indicated that the

273 expression levels of all genes were downregulated in the BT2_5 Cd treatment, including CPIP9, NFYB5,

274 and RCCR (Figure 5). The expression levels of these genes were downregulated more than 3-fold.

275 However, most candidate genes were upregulated in the BT43 Cd treatment, including NFYB5, RCCR,

276 and $\mathrm{HOX} 22$. Moreover, several genes exhibited downregulation in the BT43 treatment, including GOS9,

277 CYP71Z6, and ATP. In summary, the trend changes of most genes determined by qRT-PCR were

278 consistent with the RNA-Seq results. A few genes exhibited different trends in qRT-PCR and RNA-Seq

279 data, which may be attributed to the sensitivity of qRT-PCR.

\section{DISCUSSION}

\section{Higher Cd concentrations damage leaf tissue}

282 Toxic heavy metal contamination in plants has attracted major concern in recent years because plants,

283 including crops, can affect animal and human health (Nan et al., 2002). Cd is an unessential element for

284 plants. However, Cd can interact with other elements, such as zinc $(\mathrm{Zn})$, copper $(\mathrm{Cu})$, and manganese

285 (Mn), and affect their uptake and translocation (Lachman et al., 2015). Some studies have reported that Cd

286 can affect plant development in various plant species by inhibiting the absorption of water and nutrients, 
287 resulting in various symptoms of injury in vivo or in vitro (Li et al., 2008). Cd can interfere with several

288 photosynthetic complexes, resulting in reduced photosynthetic carbon assimilation (Maksymiec et al.,

289 2007). Moreover, Cd affects guard cell regulation via calcium channels, thus disrupting the plant's water

290 status (Perfus-Barbeoch et al., 2002). Higher Cd concentrations have been shown to reduce seed

291 germination and root and shoot elongation in Medicago sativa L. (Peralta et al., 2001). Wang et al. (2007)

292 revealed that $\mathrm{Cd}$ interacts with other metals $(\mathrm{Mn}, \mathrm{Fe}, \mathrm{Cu}$, and $\mathrm{Zn})$ that may accumulate in the roots and

293 shoots of Zea mays L. Cd caused significant inhibition of growth in Pisum sativum L. roots and leaves by

294 reducing the rate of photosynthesis and the chlorophyll content of leaves, and altering the nutrient status

295 (Sandalio et al., 2001). Cd induced production of $\mathrm{ROS}, \mathrm{H}_{2} \mathrm{O}_{2}$, and $\mathrm{O}_{2}{ }^{-}$in pea leaves, and these signal

296 molecules activate several defense genes against Cd toxicity (Romero-Puertas et al., 2004). In our study,

$2972.5 \mathrm{mM}$ (BT2_5) and $43 \mathrm{mM}$ (BT43) Cd damaged leaf tissue in bentgrass (Figure 1b, c), while plants

298 given $0 \mathrm{mM}(\mathrm{CK})$ remained healthy after 7 days. RNA-Seq data showed that higher Cd concentrations

299 affected the water uptake and nutritional status of leaves, causing a tissue morphological disorder in

300 bentgrass. These results were consistent with previous reports.

301 MAPK cascades play active roles in the response to Cd stress

302 The plant response to Cd stress involves changes in the expression patterns of many genes. To adapt to

303 difficult environmental conditions, specific genes were activated by plant cells. Signal transduction

304 pathways can drive differential gene regulation (DalCorso et al., 2010).

305 MAPK cascades occur in response to various osmotic stresses, causing hyperosmotic changes in the

306 volume and turgor pressure of the plant cell. To adapt to such stresses, plant cells produce stabilizing

307 osmolytes to increase their salt tolerance (Hanson et al., 1994). Several MAPK factors have been reported

308 in A. thaliana, including ATMAPKK1, which is upregulated under Cd stress (Suzuki et al., 2001). The

309 accumulation of ROS activates the MPK3 and MPK6 response under Cd stress (Liu et al., 2010). In Oryza

310 sativa, MAPK cascades have also been reported in response to $\mathrm{Cd}$ stress, including OsMKK4,

311 OsMSRMK2, OsMSRMK3, OsWJUMK, OsMPK2, OsMPK3, and OsMPK6 (Agrawal et al., 2002; Agrawal

312 et al., 2003; Yeh et al., 2004; Yeh et al., 2007). Moreover, SAMK, SIMK, MMK2, and MMK3 were

313 upregulated under $\mathrm{Cu} / \mathrm{Cd}$ stress in M. sativa (Jonak et al., 2004). ZmMPK3 exhibited upregulation based

314 on mRNA levels in Z. mays (Wang et al., 2010). In our study, MAPKKK3 and MAPKKK12 were

315 enhanced in creeping bentgrass in response to Cd stress. MAPKKK12 was upregulated in the BT2_5 and 
316 BT43 treatments (Table S6). MAPKKK12 may play an important role in activating tolerance to Cd stress

317 in creeping bentgrass.

318 WRKY TFs play important roles in the creeping bentgrass response to Cd stress

319 WRKY family members contain highly conserved domains, which play many roles in the regulation of

320 gene expression, including under drought, salinity, and nutrient starvation conditions (Chen et al., 2012).

321 Several WRKY TFs are important components of plant signal transduction during abiotic stress responses.

322 Overexpression of OsWRKY08 in A. thaliana resulted in increased tolerance to mannitol stress through

323 increased root production in terms of number and length (Song et al., 2009). Overexpression of

324 OsWRKY72 in A. thaliana showed that it is involved in multiple physiological processes and interferes in

325 the ABA signal and auxin transport pathway (Song et al., 2010). Three GmWRKY genes from soybean

326 were investigated in A. thaliana, and overexpression of GmWRKY21 increased tolerance to cold stress,

327 GmWRKY54 was involved in salt and drought tolerance, and GmWRKY13 increased tolerance to salt stress

328 (Zhou et al., 2008). WRKY75 was determined to regulate the nutrient starvation response in A. thaliana.

329 Our study showed that six WRKY TFs are involved in Cd stress in creeping bentgrass. WRKY23 and

330 WRKY75 were quickly upregulated from lower to higher Cd concentrations (Table S9), indicating that

331 WRKY23 and WRKY75 could increase tolerance to Cd stress. However, WRKY12, WRKY2, and WRKY53

332 were downregulated from lower to higher Cd concentrations (Table S9), suggesting negative regulatory

333 roles in the response to Cd stress.

\section{Role of bZIP TFs in the creeping bentgrass response to $\mathrm{Cd}$ stress}

335 The bZIP TFs make up a large family, of which many genes are expressed in plants under abiotic stress 336 (Hurst, 1994; Vetten et al., 1995). In A. thaliana, the TFs AtbZIP1-AtbZIP75 were reported to regulate a

337 variety of biological processes such as stress signaling, light response, and seed development. Among

338 these TFs, seven have been identified as being involved in stress signaling: AtbZIP39, AtbZIP36, 339 AtbZIP38, AtbZIP66, AtbZIP40, AtbZIP35, and AtbZIP37 (Jakoby et al., 2002; Choi et al., 2000;

340 Finkelstein and Lynch, 2000; Lopez-Molina et al., 2001; Uno et al., 2000). Overexpression of the

341 SIAREB1 gene in the bZIP family led to upregulation under salt stress in tobacco (Yánez et al., 2009). The

342 ZmbZIP72 gene from maize was overexpressed in A. thaliana, which showed that ZmbZIP72 functions as

343 an ABA-dependent TF and increases abiotic stress tolerance (Ying et al., 2012). In the present study, three

344 bZIP family genes were obtained from creeping bentgrass under Cd stress, including bZIP06, bZIP43, and 
345 bZIP19 (Table S10). bZIP43 was upregulated throughout the Cd treatment process, while bZIP06 and 346 bZIP19 genes were upregulated at the lower concentration (BT2_5) and downregulated at the higher 347 concentration (BT43). In summary, bZIP43 may play a positive regulatory role under Cd stress.

\section{ERF TFs expressed in response to Cd stress}

349 The expression of $E R F$ genes plays crucial roles in plant stress responses. For example, AtERF73/HRE1 in 350 A. thaliana plays a negative regulatory role in response to ethylene (Yang et al., 2011). Overexpression of 351 TSRF1 in the ERF family enhances osmotic and drought tolerances in rice (Quan et al., 2010, Oono et al., 352 2014). TaERF3 activates several genes related to stress that enhance the ability of wheat to adapt to salt 353 and drought stresses (Rong al., 2014). Overexpression of OsERF922 decreased tolerance to salt stress in 354 M. oryzae (Liu et al., 2012). Meanwhile, overexpression of GmERF3 increased salt and drought tolerance 355 in tobacco (Zhang et al., 2009). Moreover, overexpression of NtERF5 enhanced resistance to tobacco 356 mosaic virus (Fischer and Dröge-Laser, 2004). In summary, ERF TFs and ERF genes play important roles 357 in biological and abiotic stress responses. Our RNA-Seq data showed that seven DEGs involved in Cd 358 stress in creeping bentgrass, $E R F 1 B, E R F 110, E R F 7, E R F 113$, and $E R F 15$, were upregulated from lower 359 to higher Cd concentrations (Table S11). These ERF genes may play positive regulatory roles in the 360 response to $\mathrm{Cd}$ stress in creeping bentgrass. On the other hand, the ERF4 gene was downregulated in all 361 Cd treatments, and may play a negative role in Cd stress.

362 Roles of MYB TFs in Cd stress

363 MYB proteins have a highly conserved DNA-binding domain called the MYB domain (Martin and Paz364 Ares, 1997) and have been widely investigated in plant species such as A. thaliana, rice (O. sativa), and 365 maize (Chen et al., 2006, Oono et al.,2016, Yue et al., 2012). Several MYB TFs are involved in abiotic

366 stresses; for example, overexpression of $D w M Y B 2$ in A. thaliana suppressed iron transport from root to 367 shoot (Chen et al., 2006). The function of MxMYB1 has been identified as negative regulation of iron 368 uptake and storage in A. thaliana (Shen et al., 2008). Overexpression of MYB15 enhanced drought and salt 369 tolerance in A. thaliana (Ding et al., 2009). Overexpression of MdoMYB121 enhanced tolerances to 370 salinity and drought in tomato and apple plants (Cao et al., 2013). Overexpression of TaMYB33 and 371 TaMYB73 enhanced salt and drought tolerances (Qin et al., 2012; He et al., 2011). Moreover, 372 overexpression of $O S M Y B 48-1$ in rice promoted drought and salinity tolerance through regulation of ABA 373 synthesis (Xiong et al., 2014). In our study, four DEGs in the MYB family were enhanced under Cd stress 
374 (MYB39, MYB108, MYB305, and MYB4). All MYB TF genes were upregulated more than 2-fold in the 375 higher Cd treatment (BT43). Moreover, in BT2_5, MYB39, MYB108, and MYB305 were upregulated,

376 while MYB4 was downregulated (Table S12). Our results indicated that MYB39, MYB108, and MYB305

377 might play positive roles in the response of creeping bentgrass to $\mathrm{Cd}$ stress.

\section{CONCLUSIONS}

379 This study investigated the molecular characteristics of DEGs under Cd stress based on transcriptomic 380 analysis. A total of 463,184 unigenes were obtained from creeping bentgrass leaves. Changes in leaf tissue 381 morphology were observed, revealing that higher Cd concentrations damage leaf tissues. Moreover, four 382 key TF families (WRKY, bZIP, ERF, and MYB) were involved in Cd stress in creeping bentgrass. These 383 TFs exhibited active expression in RNA-Seq data. For example, ERF115 was upregulated more than 5384 fold. Previous research has shown that these TFs are involved in abiotic stress responses. We found that 385 these four TFs may play crucial roles in the response of creeping bentgrass to Cd stress. This study 386 provides a novel perspective for elucidating the molecular mechanisms of the response of creeping 387 bentgrass to Cd stress, and provides important reference information for phytoremediation.

\section{ACKNOWLEDGEMENTS}

389 We would like to thank Vladimir Uversky academic editor and two anonymous reviewers for their

390 comments that helped to greatly improve the manuscript.

\section{REFERENCES}

392 Agrawal G K, Rakwal R, \& Iwahashi H. 2002. Isolation of novel rice (Oryza sativa L.) multiple stress responsive MAP kinase gene, OsMSRMK2, whose mRNA accumulates rapidly in response to

Agrawal G K, Agrawal S K, Shibato J, Iwahashi H, \& Rakwal R. 2003. Novel rice MAP kinases OsMSRMK3 and OsWJUMK1 involved in encountering diverse environmental stresses and developmental regulation. Biochemical and Biophysical Research Communications 300(3): 775-783 DOI 10.1016/S0006-291X(02)02868-1.

Anders S, \& Huber W. 2010. Differential expression analysis for sequence count data. Genome Biology 
11(10): R106 DOI 10.1186/gb-2010-11-10-r106.

402 Choi H I, Hong J H, Ha J O, Kang J Y, \& Kim S Y. 2000. ABFs, a family of ABA-responsive element 403 binding factors. Journal of Biological Chemistry 275(3): 1723-1730 DOI 10.1074/jbc.275.3.1723.

404 Chen Y H, Wu X M, Ling H Q, \& Yang W C. 2006. Transgenic expression of DwMYB2 impairs iron 405 transport from root to shoot in Arabidopsis thaliana. Cell Research 16(10): 830 DOI $406 \quad 10.1038 /$ sj.cr.7310099.

407 Chen X, Wang J, Shi Y, Zhao M Q, \& Chi G Y. 2011. Effects of cadmium on growth and photosynthetic 408 activities in pakchoi and mustard. Botanical Studies $52(1)$

DOI https://ejournal.sinica.edu.tw/bbas/content/2011/1/Bot521-05.pdf.

Chen L, Song Y, Li S, Zhang L, Zou C, \& Yu D. 2012. The role of WRKY transcription factors in plant abiotic stresses. Biochimica et Biophysica Acta (BBA)-Gene Regulatory Mechanisms 19(2): 120-128 DOI 10.1016/j.bbagrm.2011.09.002.

Cao Z H, Zhang S Z, Wang R K, Zhang R F, \& Hao Y J. 2013. Genome wide analysis of the apple MYB transcription factor family allows the identification of MdoMYB121 gene conferring abiotic stress tolerance in plants. PLoS One 8(7): e69955 DOI 10.1371/journal.pone.0069955.

Ding Z, Li S, An X, Liu X, Qin H, \& Wang D. 2009. Transgenic expression of MYB15 confers enhanced sensitivity to abscisic acid and improved drought tolerance in Arabidopsis thaliana. Journal of Genetics and Genomics 36(1): 17-29 DOI 10.1016/S1673-8527(09)60003-5.

DalCorso G, Farinati S, \& Furini A. 2010. Regulatory networks of cadmium stress in plants. Plant Signaling \& Behavior 5(6): 663-667 DOI 10.4161/psb.5.6.11425.

Eulgem T, Rusht O, Schmelzer E, Hahibrock K, \& Somssich I. 1999. Early nuclear events in plant defense: rapid gene activation by WRKY transcription factors. EMBOJ 18(17): 4.

Ervin E H, Zhang X, Goatley J M, \& Askew S D. 2004. Trinexapac-ethyl, propiconazole, iron, and biostimulant effects on shaded creeping bentgrass. Hort Technology 14(4): 500-506 DOI horttech.ashspublications.org/content/14/4/500.short.

Ekblom R, \& Galindo J. 2011. Applications of next generation sequencing in molecular ecology of nonmodel organisms. Heredity 107(1): 1-15 DOI 10.1038/hdy.2010.152.

Finkelstein R R, \& Lynch T J. 2000. The Arabidopsis abscisic acid response gene ABI5 encodes a basic leucine zipper transcription factor. The Plant Cell 12(4): 599-609 DOI 10.1105/tpc.12.4.599. 
430 Fischer U, \& Dröge-Laser W. 2004. Overexpression of NtERF5, a new member of the tobacco ethylene 431 response transcription factor family enhances resistance to tobacco mosaic virus. Molecular Plant432 Microbe Interactions 17(10): 1162-1171 DOI 10.1094/MPMI.2004.17.10.1162.

433 Götz S, García-Gómez J M, Terol J, Williams T D, Nagaraj S H, Nueda M J, ... \& Conesa A. 2008. High434 throughput functional annotation and data mining with the Blast2GO suite. Nucleic Acids Research 36(10): 3420-3435 DOI 10.1093/nar/gkn176.

Grabherr M G, Haas B J, Yassour M, Levin J Z, Thompson D A, Amit I, ... \& Chen Z. 2011. Full-length transcriptome assembly from RNA-Seq data without a reference genome. Nature Biotechnology 29(7): 644 DOI 10.1038/nbt.1883.

Hoagland D R, \& Arnon D I. 1950. The water-culture method for growing plants without soil. Circular. California agricultural experiment station 347:(2nd edit)

Hanson A D, Rathinasabapathi B, Rivoal J, Burnet M, Dillon M O, \& Gage D A. 1994. Osmoprotective compounds in the Plumbaginaceae: a natural experiment in metabolic engineering of stress tolerance. Proceedings of the National Academy of Sciences 91(1): $\quad 306-310$ DOI

Hurst H C. 1994. Transcription factors. 1: bZIP proteins. Protein Profile 1(2): 123-168 DOI PMID:8528902.

Hasan S A, Fariduddin Q, Ali B, Hayat S, \& Ahmad A. 2009. Cadmium: toxicity and tolerance in plants. Journal of Environmental Biology 30(2): 165-174.

He Y, Li W, Lv J, Jia Y, Wang M, \& Xia G. 2011. Ectopic expression of a wheat MYB transcription factor gene, TaMYB73, improves salinity stress tolerance in Arabidopsis thaliana. Journal of Experimental Botany 63(3): 1511-1522 DOI 10.1093/jxb/err389.

Ishikawa S, Ishimaru Y, Igura M, Kuramata M, Abe T, Senoura T, ... \& Nakanishi H. 2012. Ion-beam irradiation, gene identification, and marker-assisted breeding in the development of low-cadmium rice. Proceedings of the National Academy of Sciences 109(47): 19166-19171 DOI 10.1073/pnas.1211132109.

Jakoby M, Weisshaar B, Dröge-Laser W, Vicente-Carbajosa J, Tiedemann J, Kroj T, \& Parcy F. 2002. bZIP transcription factors in Arabidopsis. Trends in Plant Science 7(3): 106-111 DOI 10.1016/S13601385(01)02223-3.

Jonak C, Nakagami H, \& Hirt H. 2004. Heavy metal stress. Activation of distinct mitogen-activated protein kinase pathways by copper and cadmium. Plant Physiology 136(2): 3276-3283 DOI 
10.1104/pp.104.045724.

460

461

462

463

464

465

466

467

468

469

470

471

472

473

474

475

476

477

478

479

480

481

482

483

484

485

486

487

Juwarkar A A, Yadav S K, Kumar P, \& Singh S K. 2008. Effect of biosludge and biofertilizer amendment on growth of Jatropha curcas in heavy metal contaminated soils. Environmental Monitoring and Assessment 145(1): 7-15 DOI 10.1007/s10661-007-0012-9.

Krämer U. 2005. Phytoremediation: novel approaches to cleaning up polluted soils. Current Opinion in Biotechnology 16(2): 133-141 DOI 10.1016/j.copbio.2005.02.006.

Livak K J, \& Schmittgen T D. 2001. Analysis of relative gene expression data using real-time quantitative PCR and the $2^{-\triangle \Delta C T}$ method. Methods 25(4): 402-408 DOI 10.1006/meth.2001.1262.

Lopez-Molina L, Mongrand S, \& Chua N H. 2001. A postgermination developmental arrest checkpoint is mediated by abscisic acid and requires the ABI5 transcription factor in Arabidopsis. Proceedings of the National Academy of Sciences 98(8): 4782-4787 DOI 10.1073/pnas.081594298.

Li M, Zhang L J, Tao L, \& Li W. 2008. Ecophysiological responses of Jussiaea rapens to cadmium exposure. Aquatic Botany 88(4): 347-352 DOI 10.1016/j.aquabot.2008.01.002.

Liu X M, Kim K E, Kim K C, Nguyen X C, Han H J, Jung M S, ... \& Chung W S. 2010. Cadmium activates Arabidopsis MPK3 and MPK6 via accumulation of reactive oxygen species. Phytochemistry 71(5-6): 614-618 DOI 10.1016/j.phytochem.2010.01.005.

Li B, \& Dewey C N. 2011. RSEM: accurate transcript quantification from RNA-Seq data with or without a reference genome. BMC Bioinformatics 12(1): 323 DOI 10.1186/1471-2105-12-323.

Liu D, Chen X, Liu J, Ye J, \& Guo Z. 2012. The rice ERF transcription factor OsERF922 negatively regulates resistance to Magnaporthe oryzae and salt tolerance. Journal of Experimental Botany 63(10): 3899-3911 DOI 10.1093/jxb/ers079.

Lachman J, Kotíková Z, Zámečníková B, Miholová D, Száková J, \& Vodičková H. 2015. Effect of cadmium stress on barley tissue damage and essential metal transport into plant. Open Life Sciences 10(1) DOI 10.1515/biol-2015-0004.

Martin C, \& Paz-Ares J. 1997. MYB transcription factors in plants. Trends in Genetics 13(2): 67-73 DOI 10.1016/S0168-9525(96)10049-4.

Maksymiec W, Wojcik M, \& Krupa Z. 2007. Variation in oxidative stress and photochemical activity in Arabidopsis thaliana leaves subjected to cadmium and excess copper in the presence or absence of jasmonate and ascorbate. Chemosphere 66(3): 421-427 DOI 10.1016/j.chemosphere.2006.06.025. 
488

490

Miyadate H, Adachi S, Hiraizumi A, Tezuka K, Nakazawa N, Kawamoto T, ... \& Satoh-Nagasawa N. 2011. OsHMA3, a P1B-type of ATPase affects root-to-shoot cadmium translocation in rice by mediating efflux into vacuoles. New Phytologist 189(1): 190-199 DOI 10.1111/j.1469-8137.2010.03459.x.

Nan Z, Li J, Zhang J, \& Cheng G. 2002. Cadmium and zinc interactions and their transfer in soil-crop system under actual field conditions. Science of the Total Environment 285(1-3): 187-195 DOI 10.1016/S00489697(01)00919-6.

Nawrot T S, Plusquin M, Hogervorst J G, Roels H, Celis H, Thijs L, ... \& Staessen J A. 2006. Environmental exposure to cadmium and risk of cancer: a prospective population-based study. Lancet Oncology 7(2): 119-126 DOI 10.1016/S1470-2045(06)70545-9.

Oono Y, Yazawa T, Kawahara Y, Kanamori H, Kobayashi F, Sasaki H, ... \& Matsumoto T. 2014. Genomewide transcriptome analysis reveals that cadmium stress signaling controls the expression of genes in drought stress signal pathways in rice. PLoS One 9(5): e96946 DOI : 10.1371/journal.pone.0096946.

Oono Y, Yazawa T, Kanamori H, Sasaki H, Mori S, Handa H, \& Matsumoto T. 2016. Genome-wide transcriptome analysis of cadmium stress in rice. BioMed research international :2016 DOI:10.1155/2016/9739505.

Peralta J R, Gardea-Torresdey J L, Tiemann K J, Gomez E, Arteaga S, Rascon E, \& Parsons J G. 2001. Uptake and effects of five heavy metals on seed germination and plant growth in alfalfa (Medicago sativa L.). Bulletin of Environmental Contamination and Toxicology 66(6): 727-734 DOI 10.1007/s001280069.

Perfus-Barbeoch L, Leonhardt N, Vavasseur A, \& Forestier C. 2002. Heavy metal toxicity: cadmium permeates through calcium channels and disturbs the plant water status. The Plant Journal 32(4): 539-548 DOI 10.1046/j.1365-313X.2002.01442.x.

Quan R, Hu S, Zhang Z, Zhang H, Zhang Z, \& Huang R. 2010. Overexpression of an ERF transcription factor TSRF1 improves rice drought tolerance. Plant Biotechnology Journal 8(4): 476-488 DOI 10.1111/j.1467-7652.2009.00492.x.

Qin Y, Wang M, Tian Y, He W, Han L, \& Xia G. 2012. Over-expression of TaMYB33 encoding a novel wheat MYB transcription factor increases salt and drought tolerance in Arabidopsis. Molecular Biology Reports 39(6): 7183-7192 DOI 10.1007/s11033-012-1550-y.

Romero-Puertas M C, Rodríguez-Serrano M, Corpas F J, Gomez M D, Del Rio L A, \& Sandalio L M. 2004. Cadmium-induced subcellular accumulation of $\mathrm{O}_{2}^{-{ }^{-}}$and $\mathrm{H}_{2} \mathrm{O}_{2}$ in pea leaves. Plant, Cell \& 
518 Rong W, Qi L, Wang A, Ye X, Du L, Liang H, ... \& Zhang Z. 2014. The ERF transcription factor TaERF3 promotes tolerance to salt and drought stresses in wheat. Plant Biotechnology Journal 12(4): 468-479 DOI 10.1111/pbi.12153.

Snyder V. 1972. Nitrogen and chelated iron fertilization on the growth and physiology of creeping bentgrass (Agrostis palustrus Huds) (Doctoral dissertation, Virginia Polytechnic Institute and State University).

Salt D E, Smith R D, \& Raskin I. 1998. Phytoremediation. Annual Review of Plant Biology 49(1): 643-668 DOI 10.1146/annurev.arplant.49.1.643.

Sandalio L M, Dalurzo H C, Gomez M, Romero-Puertas M C, \& Del Rio L A. 2001. Cadmium-induced changes in the growth and oxidative metabolism of pea plants. Journal of Experimental Botany 52(364):

Suzuki N, Koizumi N, \& Sano H. 2001. Screening of cadmium-responsive genes in Arabidopsis thaliana. Plant, Cell \& Environment 24(11): 1177-1188 DOI 10.1046/j.1365-3040.2001.00773.x.

Shen J, Xu X, Li T, Cao D, \& Han Z. 2008. An MYB transcription factor from Malus xiaojinensis has a potential role in iron nutrition. Journal of Integrative Plant Biology 50(10): 1300-1306 DOI

Song Y, Jing S, \& Yu D. 2009. Overexpression of the stress-induced OsWRKY08 improves osmotic stress tolerance in Arabidopsis. Chinese Science Bulletin 54(24): 4671-4678 DOI 10.1007/s11434-009-0710-5.

Song Y, Chen L, Zhang L, \& Yu D. 2010. Overexpression of OsWRKY72 gene interferes in the abscisic acid signal and auxin transport pathway of Arabidopsis. Journal of Biosciences 35(3): 459-471 DOI 10.1007/s12038-010-0051-1.

Satoh-Nagasawa N, Mori M, Nakazawa N, Kawamoto T, Nagato Y, Sakurai K, ... \& Akagi H. 2011. Mutations in rice (Oryza sativa) heavy metal ATPase 2 (OsHMA2) restrict the translocation of zinc and cadmium. Plant and Cell Physiology 53(1): 213-224 DOI 10.1093/pcp/pcr166.

Tang W, Charles T M, \& Newton R J. 2005. Overexpression of the pepper transcription factor CaPF1 in transgenic Virginia pine (Pinus virginiana Mill.) confers multiple stress tolerance and enhances organ growth. Plant Molecular Biology 59(4): 603-617 DOI 10.1007/s11103-005-0451-z.

\section{Trapnell C, Williams B A, Pertea G, Mortazavi A, Kwan G, Van Baren M J, ... \& Pachter L. 2010.} Transcript assembly and quantification by RNA-Seq reveals unannotated transcripts and isoform 
switching during cell differentiation. Nature Biotechnology 28(5): 511-515 DOI 10.1038/nbt.1621.

547

548

Takahashi R, Ishimaru Y, Senoura T, Shimo H, Ishikawa S, Arao T, ... \& Nishizawa N K. 2011. The OSNRAMP1 iron transporter is involved in Cd accumulation in rice. Journal of Experimental Botany 62(14): 4843-4850 DOI 10.1093/jxb/err136.

Uno Y, Furihata T, Abe H, Yoshida R, Shinozaki K, \& Yamaguchi-Shinozaki K. 2000. Arabidopsis basic leucine zipper transcription factors involved in an abscisic acid-dependent signal transduction pathway under drought and high-salinity conditions. Proceedings of the National Academy of Sciences 97(21): 11632-11637 DOI 10.1073/pnas.190309197.

Ueno D, Yamaji N, Kono I, Huang C F, Ando T, Yano M, \& Ma J F. 2010. Gene limiting cadmium accumulation in rice. Proceedings of the National Academy of Sciences 107(38): 16500-16505 DOI 10.1073/pnas.1005396107.

Vargas J M. 1993. Management of turfgrass diseases. CRC Press.

Vetten N C, \& Ferl R J. 1995. Characterization of a maize G-box binding factor that is induced by hypoxia. The Plant Journal 7(4): 589-601 DOI 10.1046/j.1365-313X.1995.7040589.x.

Vig K, Megharaj M, Sethunathan N, \& Naidu R. 2003. Bioavailability and toxicity of cadmium to microorganisms and their activities in soil: a review. Advances in Environmental Research 8(1): 121-135 DOI 10.1016/S1093-0191(02)00135-1.

van de Mortel J E, Schat H, Moerland P D, van Themaat E V L, Van Der Ent S J O E R D, Blankestijn H, ... \& AARTS M G. 2008. Expression differences for genes involved in lignin, glutathione and sulphate metabolism in response to cadmium in Arabidopsis thaliana and the related $\mathrm{Zn} / \mathrm{Cd}$-hyperaccumulator Thlaspi caerulescens. Plant, Cell \& Environment 31(3): 301-324 DOI 10.1111/j.1365-3040.2007.01764.x.

Wang M, Zou J, Duan X, Jiang W, \& Liu, D. 2007. Cadmium accumulation and its effects on metal uptake in maize (Zea mays L.). Bioresource Technology 98(1): 82-88 DOI 10.1016/j.biortech.2005.11.028.

Wei W, Zhang Y, Han L, Guan Z, \& Chai T. 2008. A novel WRKY transcriptional factor from Thlaspi caerulescens negatively regulates the osmotic stress tolerance of transgenic tobacco. Plant Cell Reports 27(4): 795-803 DOI 10.1007/s00299-007-0499-0.

Wang Z, Gerstein M, \& Snyder M. 2009. RNA-Seq: a revolutionary tool for transcriptomics. Nature reviews genetics 10(1): 57-63 DOI 10.1038/nrg2484.

Wang J, Ding H, Zhang A, Ma F, Cao J, \& Jiang M. 2010. A novel mitogen-activated protein kinase gene in 
575

576

577

578

579

580

581

582

583

584

585

586

587

588

589

590

591

592

593

594

595

596

597

598

599

600

601

602

603

maize (Zea mays), ZmMPK3, is involved in response to diverse environmental cues. Journal of Integrative Plant Biology 52(5): 442-452 DOI 10.1111/j.1744-7909.2010.00906.x.

Xiong H, Li J, Liu P, Duan J, Zhao Y, Guo X, ... \& Li Z. 2014. Overexpression of OsMYB48-1, a novel MYB-related transcription factor, enhances drought and salinity tolerance in rice. PLoS One 9(3): e92913 DOI 10.1371/journal.pone.0092913.

Xu L, Wang Y, Liu W, Wang J, Zhu X, Zhang K, ... \& Gong Y. 2015. De novo sequencing of root transcriptome reveals complex cadmium-responsive regulatory networks in radish (Raphanus sativus L.). Plant Science 236: 313-323 DOI 10.1016/j.plantsci.2015.04.015.

Yeh C M, Hsiao L J, \& Huang H J. 2004. Cadmium activates a mitogen-activated protein kinase gene and MBP kinases in rice. Plant and Cell Physiology 45(9): 1306-1312 DOI 10.1093/pcp/pch135.

Yeh C M, Chien P S, \& Huang H J. 2007. Distinct signalling pathways for induction of MAP kinase activities by cadmium and copper in rice roots. Journal of Experimental Botany 58(3): 659-671 DOI 10.1093/jxb/erl240.

Yánez M, Cáceres S, Orellana S, Bastías A, Verdugo I, Ruiz-Lara S, \& Casaretto J A. 2009. An abiotic stress-responsive bZIP transcription factor from wild and cultivated tomatoes regulates stress-related genes. Plant Cell Reports 28(10): 1497-1507 DOI 10.1007/s00299-009-0749-4.

Yang C Y, Hsu F C, Li J P, Wang $\mathbf{N}$ N, \& Shih M C. 2011. The AP2/ERF transcription factor AtERF73/HRE1 modulates ethylene responses during hypoxia in Arabidopsis. Plant Physiology 156(1): 202-212 DOI 10.1104/pp.111.172486.

Ying S, Zhang D F, Fu J, Shi Y S, Song Y C, Wang T Y, \& Li Y. 2012. Cloning and characterization of a maize bZIP transcription factor, ZmbZIP72, confers drought and salt tolerance in transgenic Arabidopsis. Planta 235(2): 253-266 DOI 10.1007/s00425-011-1496-7.

Yue R, Lu C, Qi J, Han X, Yan S, Guo S, ... \& Chi H. 2016. Transcriptome analysis of cadmium-treated roots in maize (Zea mays L.). Frontiers in plant science 7: 1298 DOI:10.3389/fpls.2016.01298.

Yu R, Li D, Du X, Xia S, Liu C, \& Shi G. 2017. Comparative transcriptome analysis reveals key cadmium transport-related genes in roots of two pak choi (Brassica rapa L. ssp. chinensis) cultivars. BMC Genomics 18(1): 587 DOI 10.1186/s12864-017-3973-2.

Zhou Q Y, Tian A G, Zou H F, Xie Z M, Lei G, Huang J, ... \& Chen S Y. 2008. Soybean WRKY-type transcription factor genes, GmWRKY13, GmWRKY21 and GmWRKY54 confer differential tolerance to 
604 abiotic stresses in transgenic Arabidopsis plants. Plant Biotechnology Journal 6(5): 486-503 DOI $605 \quad 10.1111 / j .1467-7652.2008 .00336 . x$.

606 Zhang G, Chen M, Li L, Xu Z, Chen X, Guo J, \& Ma Y. 2009. Overexpression of the soybean GmERF3 607 gene, an AP2/ERF type transcription factor for increased tolerances to salt, drought, and diseases in 608 transgenic tobacco. Journal of Experimental Botany 60(13): 3781-3796 DOI 10.1093/jxb/erp214. 
Figure 1

Figure 1: Leaf tissue morphology.

a. CK; b. BT_2.5; c. BT43, bar $=50 \mu \mathrm{m}$, the leaf tissue stained with saffron and fast green FCF, CK cells represent morphologically, BT_2.5 cells represent minor damage, BT_43 cells represent severe damage.

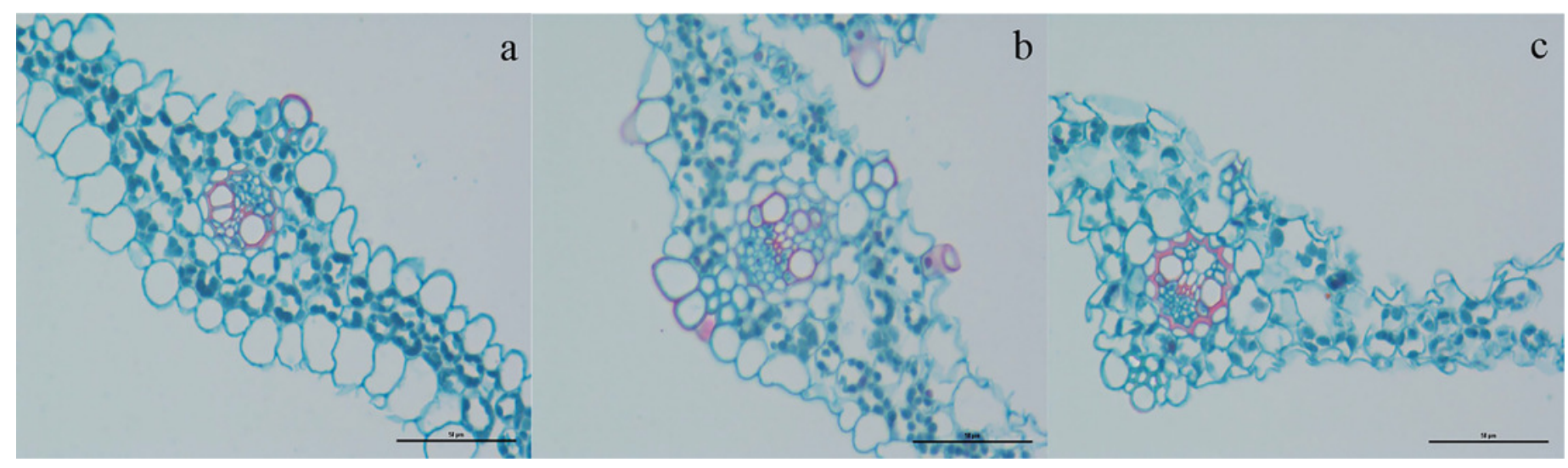




\section{Figure 2}

Figure 2: Sequence distribution of transcripts and unigenes.

a. transcript length; b. unigene length. N50: splicing transcripts in descending order of length, accumulating transcript lengths, to a length of not less than $50 \%$ of the total spliced transcripts; N90: $90 \%$ of the total length of the spliced transcript length.
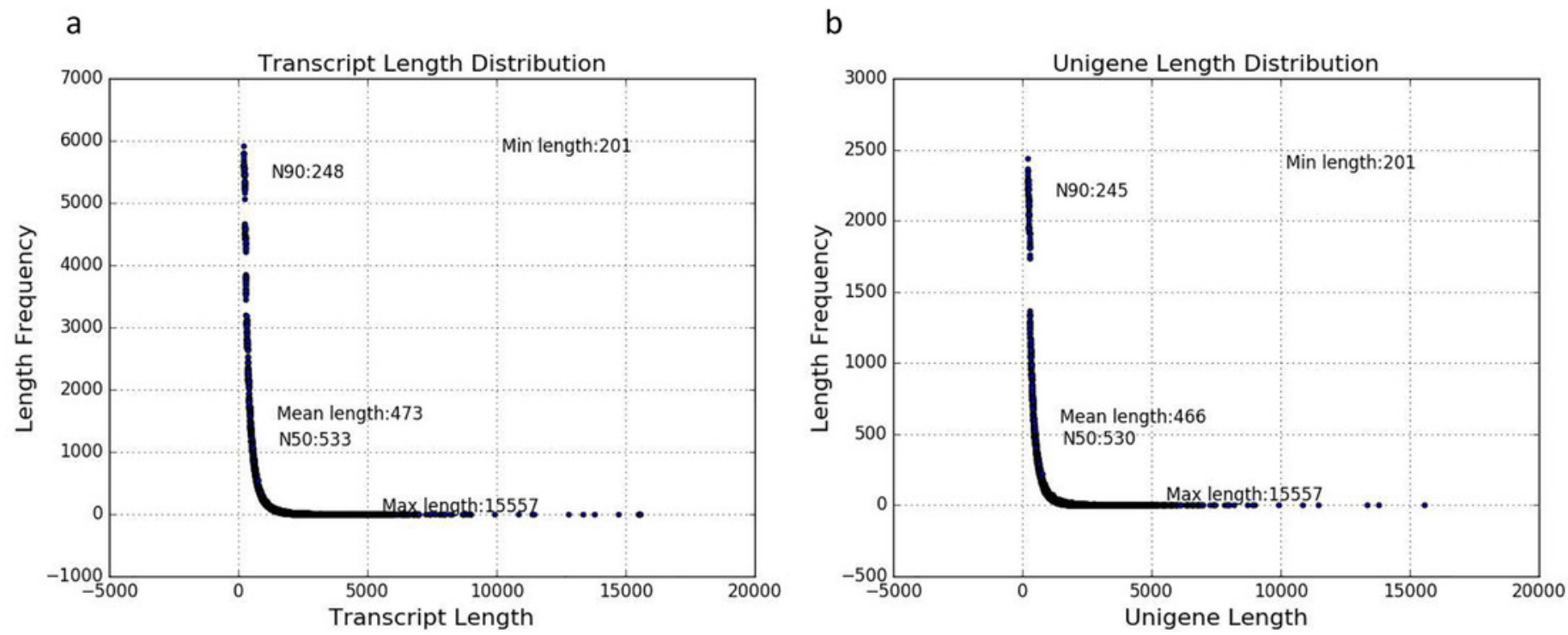


\section{Figure 3}

Figure 3: KEGG classifications.

\section{A-E for Cellular Processes, Environmental Information Processing, Genetic Information}

Processing, Metabolism; and Organismal Systems.

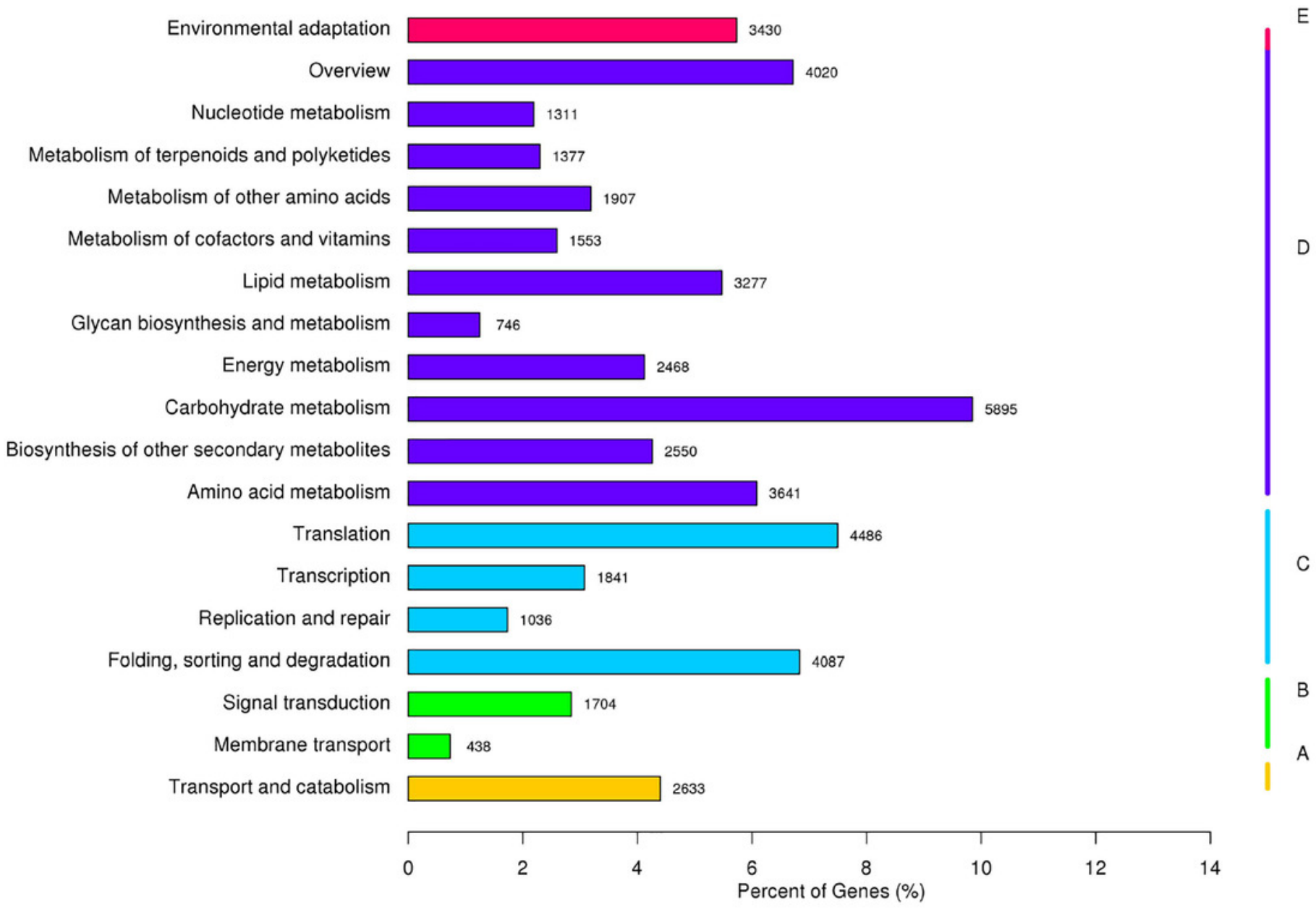




\section{Figure 4}

Figure 4: Volcano plots comparing differentially expressed genes (DEGs).

a. DEGs between BT2_5 and CK; b. DEGs between BT43 and BT2_5; c. DEGs between BT43 and CK. Red dots represent up-regulated genes; Green dots represent down-regulated genes; Blue dots represent genes that have not been changed.
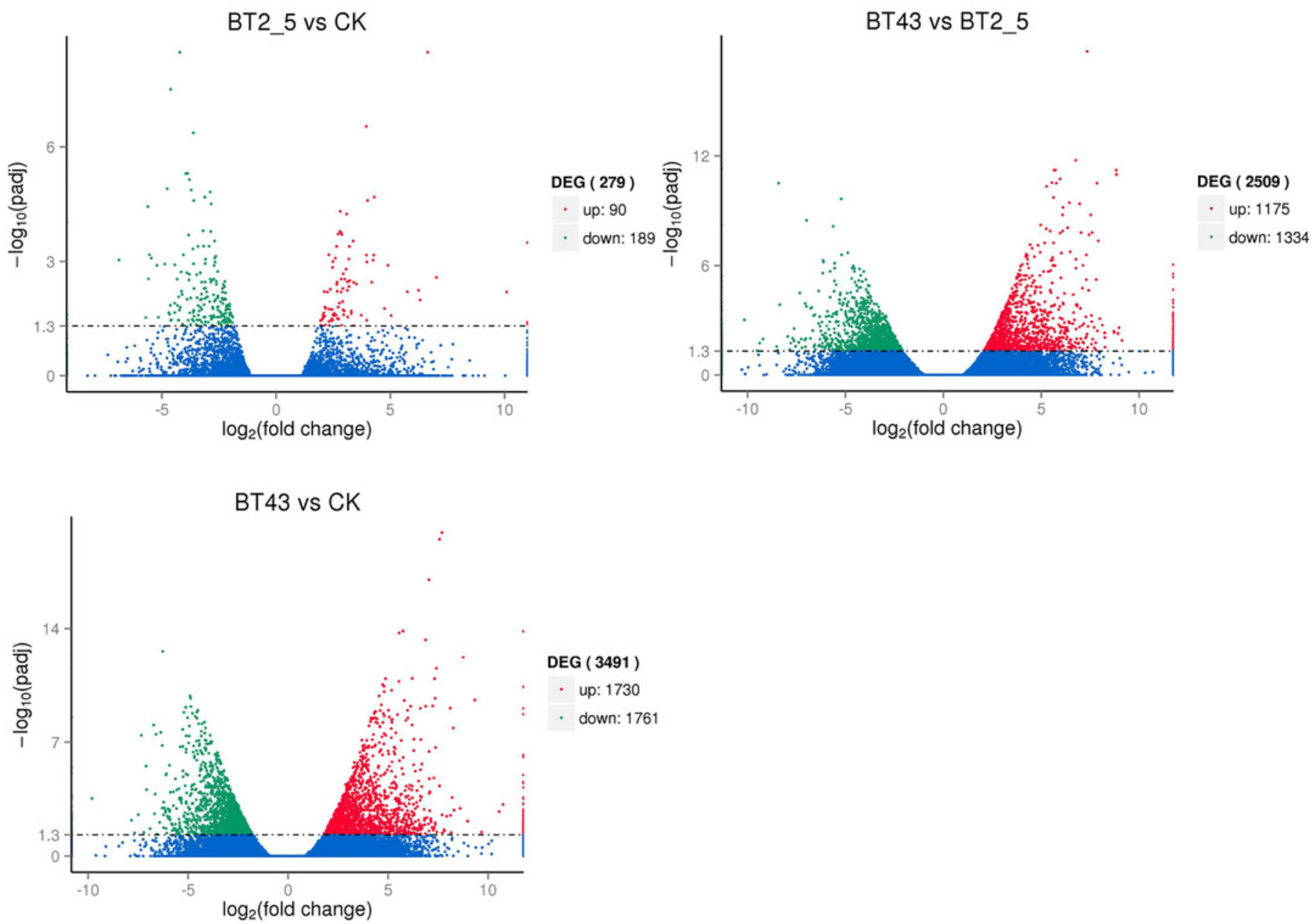
Figure 5

Figure 5: Validation of the selected DEGs using qRT-PCR under CK, BT2_5, and BT43 Cd treatment conditions.

The relative expression of each gene was determined from three technical replicates using the $2^{-\Delta \Delta \mathrm{ct}}$ method.
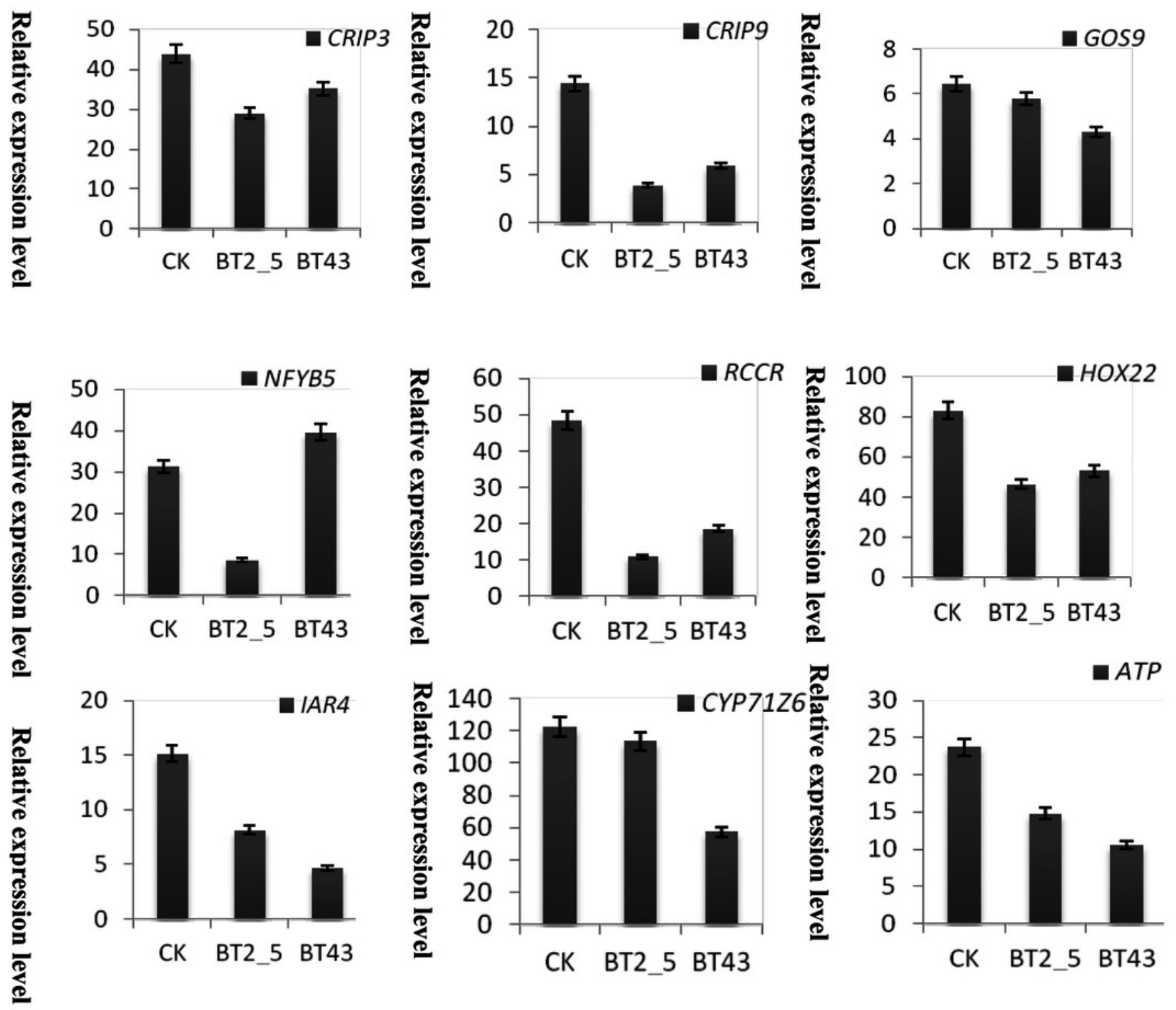


\section{Table $\mathbf{1}$ (on next page)}

Table1: RNA sequencing data quality profile.

Q20, bases with Phred scores greater than 20 as a percentage of total bases; Q30, bases with Phred scores greater than 30 as a percentage of total bases; and GC, G+C content. 
1

\begin{tabular}{cccccccc}
\hline Sample & Raw Reads & Clean reads & Clean bases & Error (\%) & $\begin{array}{c}\text { Q20 } \\
(\%)\end{array}$ & Q30 (\%) & $\begin{array}{c}\text { GC } \\
(\%)\end{array}$ \\
\hline CK & $51,167,908$ & $49,565,106$ & $7.43 \mathrm{G}$ & 0.02 & 95.91 & 89.99 & 57.82 \\
BT_2.5 & $55,312,134$ & $53,203,108$ & $7.98 \mathrm{G}$ & 0.01 & 97.63 & 93.75 & 57.71 \\
BT43 & $52,608,602$ & $50,981,914$ & $7.65 \mathrm{G}$ & 0.02 & 96.07 & 90.35 & 58.34 \\
\hline
\end{tabular}




\section{Table 2 (on next page)}

Table 2: Gene annotation statistics. 
1

\begin{tabular}{ccc}
\hline & Number of Unigenes & Percentage (\%) \\
\hline Annotated in NR & 203561 & 43.94 \\
Annotated in NT & 167084 & 36.07 \\
Annotated in KO & 59855 & 12.92 \\
Annotated in SwissProt & 111508 & 24.07 \\
Annotated in PFAM & 123967 & 26.76 \\
Annotated in GO & 127047 & 27.42 \\
Annotated in KOG & 27394 & 5.91 \\
Annotated in all Databases & 14496 & 3.12 \\
Annotated in at least one Database & 260356 & 56.21 \\
Total Unigenes & 463184 & 100 \\
\hline
\end{tabular}

